\title{
The Autism-Spectrum Quotient (AQ) in Japan: A Cross-Cultural Comparison
}

\author{
Akio Wakabayashi, ${ }^{1,4}$ Simon Baron-Cohen, ${ }^{2}$ Sally Wheelwright, ${ }^{2}$ and Yoshikuni Tojo ${ }^{3}$
}

\begin{abstract}
The AQ (Autism-Spectrum Quotient) is a self-administered instrument for measuring the degree to which an adult with normal intelligence has the traits associated with the autistic spectrum. The AQ was administered in Japan to test whether the UK results would generalize to a very different culture. Three groups of subjects, adults with AS or HFA $(n=57)$, adult controls $(n=194)$, and University students $(n=1050)$ were assessed. The adults with AS/HFA had a mean AQ score which was significantly higher than both the controls and the University students. Among the controls, males scored significantly higher than females. The similarity of results in both the general population and the clinical group across the two cultures was remarkable.
\end{abstract}

KEY WORDS: Autism spectrum quotient; Asperger syndrome; high-functioning autism; normal intelligence; adults with autism; self-administered.

\section{INTRODUCTION}

Autism is defined in terms of abnormalities in social and communication development, in the presence of marked repetitive behaviour and limited imagination (APA, 1994). Asperger Syndrome (AS) is defined in terms of the individual meeting the same criteria for autism but with no history of cognitive or language delay, and not meeting the criteria for other PDD (WHO, 1992). Recently, an assumption was proposed that autism and AS lie on a continuum not just with each other, but with normality (Baron-

\footnotetext{
${ }^{1}$ Department of Psychology, Chiba University, Yayoi-cho, Inage, Chiba, Japan.

${ }^{2}$ Departments of Psychiatry, Autism Research Centre, University of Cambridge, Cambridge, UK.

${ }^{3}$ Section of Education for Children with Autism, The National Institute of Special Education, Midoricho, Musashino, Tokyo, Japan.

${ }^{4}$ Correspondence should be addressed to: Akio Wakabayashi, Department of Psychology, Chiba University, 1-33 Yayoi-cho, Inage, Chiba 263-8522, Japan; e-mail: akiowcam@mac.com
}

Cohen, 1995; Frith, 1991; Wing, 1981, 1988). The continuum view allows a quantitative approach to autism and AS which complements categorical diagnosis.

To fill the need for a short, self-administered scale for identifying the degree to which any individual adult of normal IQ may have "autistic traits", or what has been called ,the broader phenotype' (Bailey et al., 1995), we developed the Autism-Spectrum Quotient (AQ) (Baron-Cohen, Wheelwright, Skinner, Martin, \& Clubley, 2001). This was needed for both scientific reasons (e.g., establishing who is "affected" and who is not, or the degree of "caseness" of an individual, in scientific comparisons) and potentially for applied reasons (e.g., screening for possibly "affected" individuals to assist in making referrals for a full diagnostic assessment).

The AQ comprises 50 questions, made up of 10 questions assessing 5 different areas: social skill (for example: "I prefer to do things with others rather than on my own."--reversal item); attention switching (for example: "I prefer to do things the same way 
over and over again"); attention to detail (for example: "I often notice small sounds when others do not."); communication (for example: "Other people frequently tell me that what I've said is impolite, even though I think it is polite."); imagination (for example: "When I'm reading a story, I can easily imagine what the characters might look like"-reversal item). Each of the items scores 1 point if the respondent records the abnormal or autistic-like behaviour either mildly or strongly (Abnormality $=$ poor social skill, poor communication skill, poor imagination, exceptional attention to detail, poor attention-switching/strong focus of attention). Approximately half the items are worded to produce a "disagree" response, and half an "agree" response, in a high scoring person with Asperger Syndrome (AS) or high functioning autism (HFA). This is to avoid a response bias either way. Items are randomized with respect to both the expected response from a high-scorer, and their domain. The items of the AQ were selected from the domains in the "triad" of autistic symptoms (APA, 1994; Rutter, 1978; Wing \& Gould, 1979), and from demonstrated areas of cognitive abnormality in autism (Baron-Cohen et al., 2001). The final version of the AQ has a forced choice format, can be self-administered, and is straightforward to score since it does not depend on any interpretation in the scoring.

In the first study with the AQ (Baron-Cohen et al., 2001), carried out in the UK, four groups of subjects were tested: 58 adults with AS/HFA, 174 adults selected at random, 840 students in Cambridge University, and 16 winners of the UK Mathematics Olympiad. The UK study found, as predicted, that the AS/HFA group scored higher than controls, and that control males scored significantly higher than control females. There was no difference between mean AQ scores of males and females with AS/HFA. The AQ scores for student volunteers also revealed that the science students scored higher than both humanities and social science students, who did not differ from each other.

Using the rule that a useful cut-off would discriminate the groups with as many true positives and as few false positives as possible, an AQ score of $32+$ was chosen, since $79.3 \%$ of the AS/HFA group scored at this level, whilst only $2 \%$ of controls did so. Thirty-two and over also seems to be a useful cut-off for distinguishing females with AS/HFA (92.3\% scoring at this point or above) vs. control females ( $1 \%$ of whom score at this point or above). The aim of the study reported in the present paper was to replicate the UK study in a Japanese sample, using a similar methodology. This would establish whether the AQ is culture-specific (i.e., western) or cultureindependent.

\section{METHOD}

\section{Subjects}

In the Japanese study, we tested 3 groups of subjects: Group 1 comprised $n=57$ adults with AS/ HFA (44 males, 13 females). This sex ratio of 3.5:1 is similar to that the sample of the AQ study in UK. Their mean age was 26.9 years $(S D=7.88$; range: 18 57). All of these individuals had been diagnosed by psychiatrists or psychologists using DSM-IV criteria for autism or Asperger syndrome. The diagnosis for most of the AS/HFA subjects was confirmed by checking the clinical reports, or in some case from parental report. They were recruited via several sources, including the Japanese Autistic Society, specialist clinics carrying out diagnostic assessment, and some self-help groups. They are assumed to have an IQ in the normal range because all of them had completed senior-high school, and some of them had completed a university degree. Group 2 comprised $n=194$ adults selected at random (103 males, 91 females) from a general population. They were recruited through companies that were all willing to cooperate in this study. The AQ was sent to 500 employees randomly and the completed AQ was sent back to the research team, resulting in a response rate of $39 \%$. Their mean age was 33.6 years $(S D=6.2$; range: 22-56). Finally, Group 3 comprised $n=1050$ students in 5 universities in or near the Tokyo area. These were 555 males, 495 females. As a group, their mean age was $20.3(\mathrm{SD}=1.9$; range: $18-41)$.

\section{Item Translation}

The item translation proceeded as follows: first, Japanese authors translated items of the AQ (in English) into Japanese. Then the Japanese items were checked by an English-Japanese bilingual psychologist for whether they correspond with the original English items. Finally, the Japanese items were backtranslated into English by English-native speakers who could understood Japanese, and they were checked for whether they corresponded with the original English items. The final version of the Japanese AQ is similar to the original English version, an has the same forced choice format. 


\section{Procedure}

This exactly followed the procedures described in the UK study. Subjects in Groups 1 and 2 were sent the Japanese AQ by post, and the subject was instructed to complete it as quickly as possible to avoid thinking about responses too long, and to complete it on their own. Subjects in Group 2 had the option to complete this anonymously or not. Subjects in Group 3 filled out the AQ, mostly in group settings, on five occasions during introductory classes on psychology. In a few cases, students completed the AQ individually.

\section{RESULTS}

\section{AS/HFA vs. Controls, and Sex Difference}

The mean total AQ scores from each group are displayed in Table I, and subscale scores are shown in Table II. Using an ANOVA of total AQ Score by Group, there was a main effect of Group ( $F$ (2, $1301)=211.177, p<0.0001)$, with post hoc Sheffe tests revealing that Group $1>$ Group $2 \& 3(p<0.00001)$. The AS/HFA group scored a mean of 37.9 $(S D=5.31)$, which was significantly higher than both the general population group $(M=18.5, S D=6.21)$ and the student group $(M=20.7, S D=6.38)$. In the

Table I. Mean AQ Scores (and $S D$ s) by Group in Japanese Sample and the UK Study (Baron-Cohen et al., 2001)

\begin{tabular}{llcr}
\hline Group & & $\begin{array}{c}\text { Total AQ in } \\
\text { Japanese sample }\end{array}$ & $\begin{array}{c}\text { Total AQ in } \\
\text { the UK sample }\end{array}$ \\
\hline Group 1 & & & \\
AS/HFA & $M$ & 37.9 & 35.8 \\
Males & $S D$ & 5.31 & 6.5 \\
& $M$ & 37.2 & 35.1 \\
Females & $S D$ & 5.64 & 6.9 \\
& $M$ & 40.3 & 38.1 \\
Group 2 & $S D$ & 3.91 & 4.4 \\
Controls & $M$ & 18.5 & 16.4 \\
& $S D$ & 6.21 & 6.3 \\
Males & $M$ & 19.1 & 17.8 \\
& $S D$ & 5.98 & 6.8 \\
Females & $M$ & 17.9 & 15.4 \\
& $S D$ & 6.44 & 5.7 \\
Group 3 & & & \\
Students & $M$ & 20.7 & 17.6 \\
Males & $S D$ & 6.38 & 6.4 \\
& $M$ & 21.5 & 6.6 \\
Females & $S D$ & 6.29 & 6.1 \\
& $M$ & 19.9 & \\
\hline
\end{tabular}

Japanese sample the females with AS/HFA appeared to have higher AQs than the males with AS/HFA (a pattern seen in the UK sample too), but $t$-tests revealed that this was not a statistically significant difference. In both the general population (males $M=19.1, S D=5.98$; females $M=17.9, S D=6.44$ ) and the student population (males $M=21.5$, $S D=6.29$, females $M=19.9, S D=6.38$ ), there was a significant sex difference, males scoring higher than females $(t=1.508, \quad \mathrm{df}=192, \quad p<0.10 ; \quad t=4.096$, $\mathrm{df}=1048, p<0.01$, respectively). All mean subcategory AQ scores in the AS/HFA group were significantly higher than both the general population group and the student group $(t$-tests, all $p<.001)$.

\section{Test-Retest Reliability}

To confirm test-retest reliability, 54 students from Group 3 were asked to complete a second AQ 2-3 weeks after the first administration. Scores from the first and second AQ's did not differ statistically $(t=0.237, \mathrm{df}=53, n . s$.$) and were strongly correlated$ $(r=0.87, p=0.001)$.

\section{Self vs. Parent Report}

To test whether self-report by adults with AS/ HFA might be leading to incorrect scores, all subjects in Group 1 were asked if a parent could also complete an AQ on them. Thirty-two of these families agreed to do this. The parent version of the AQ omitted 10 items out of 50 (item 3, 5, 6, 8, 12, 20, 23, 27, 36, and 42 ), since these items could only be answered subjectively. The mean difference in AQ score between selfreport and parent-report for the 40-item AQ (by 32 $\mathrm{AS} / \mathrm{HFA}$ and parent pairs) was 2.1 points $(\mathrm{SD}=0.5)$, parents scoring their child more highly than their child's self-report. Correlation between AQ score by parent-report and self-report was $r=0.71(\mathrm{df}=30$, $p<.01)$.

\section{Item Analysis}

Adults with AS/HFA scored higher than controls on all items except two (item 23: "I notice patterns in things all the time.", and 29: "I am not very good at remembering phone numbers."), which controls scored higher than did the AS/HFA group. Since item 29 was also scored higher by the control groups in the UK study, compared to the group with AS/HFA, this suggests that this item is not valid to discriminate people with AS/HFA from people 
Table II. Mean AQ and Subscale Scores (and SDs) by Group in Japanese Sample

\begin{tabular}{|c|c|c|c|c|c|c|}
\hline & & Social skill & Attention switching & Local details & Communication & Imagination \\
\hline \multicolumn{7}{|l|}{ Group 1} \\
\hline \multirow[t]{2}{*}{ AS/HFA $(n=57)$} & $M$ & 8.3 & 7.9 & 6.2 & 8.2 & 7.4 \\
\hline & $S D$ & 1.80 & 1.43 & 2.22 & 1.28 & 1.63 \\
\hline \multicolumn{7}{|l|}{ Group 2} \\
\hline \multirow{2}{*}{ Controls $(n=194)$} & $M$ & 3.4 & 4.3 & 4.9 & 2.8 & 3.2 \\
\hline & $S D$ & 2.38 & 2.06 & 1.96 & 2.07 & 1.67 \\
\hline \multirow[t]{2}{*}{ Males $(n=103)$} & $M$ & 3.7 & 4.2 & 4.7 & 3.0 & 3.4 \\
\hline & $S D$ & 2.46 & 2.04 & 1.91 & 2.09 & 1.67 \\
\hline \multirow[t]{2}{*}{ Females $(n=91)$} & $M$ & 3.1 & 4.3 & 5.0 & 2.6 & 2.9 \\
\hline & $S D$ & 2.29 & 2.11 & 2.02 & 2.04 & 1.65 \\
\hline \multicolumn{7}{|l|}{ Group 3} \\
\hline \multirow[t]{2}{*}{ Students $(n=1050)$} & $M$ & 3.9 & 5.2 & 4.8 & 3.7 & 3.2 \\
\hline & $S D$ & 2.60 & 2.01 & 1.95 & 2.08 & 1.78 \\
\hline \multirow[t]{2}{*}{ Males $(n=555)$} & $M$ & 4.4 & 5.3 & 4.8 & 3.8 & 3.5 \\
\hline & $S D$ & 2.57 & 1.96 & 1.96 & 2.11 & 1.86 \\
\hline \multirow[t]{2}{*}{ Females $(n=495)$} & $M$ & 3.7 & 5.1 & 4.8 & 3.5 & 2.8 \\
\hline & $S D$ & 2.64 & 2.06 & 1.95 & 2.04 & 1.60 \\
\hline
\end{tabular}

without AS/HFA. There is an argument therefore for removing this item from a revised AQ. However, these two items were conservatively retained in the analysis here since they served to reduce the size of group differences, and to leave the AQ directly comparable with the original UK study.

\section{Internal Consistency}

The internal consistency of the Japanese AQ was calculated only using the data of control groups, because the AS/HFA group (who tended to agree on most items) would risk inflating the internal consistency. Cronbach's $\alpha$ Coefficients were as follows. AQ total $=0.81$, Communication $=0.64 ;$ Social skill $=$ 0.78 ; Imagination $=0.51$; Local details $=0.57$; Attention switching $=0.63$. These indicate that the internal consistency of the total Japanese AQ scale is high, and the subscales vary in internal consistency from moderate to high.

\section{Determining a Cut-off Point}

Percentage of each group scoring at or above each Japanese AQ score is shown in Table III. Using the same rule from the UK study, that a useful cut-off would discriminate the groups with as many true positives and as few false negatives as possible, as Japanese AQ score of $33+$ was chosen, since $87.8 \%$ of the AS/HFA group scored at this level, whilst only $3 \%$ of controls did so. However, with regard to determine a caseness cut-off point for a question- naire, it is important to examine the false positive and false negative rate of the questionnaire when applied to a sample that includes clearly defined cases and non-cases of a specific disorder. In this study the diagnostic status of people in the control groups is not known. It is possible (assuming a rate of autism spectrum conditions of about 1 in 200), that there were some cases of AS/HFA among both the general population group and the student group. These may have not had a diagnosis. If anything, this should serve to boost the score of AQ in control groups. This means that the cut-off point that we proposed here should be somewhat higher than that would be chosen from a control subjects of people without AS/ HFA.

\section{Scientists vs. Non-Scientists}

Table IV shows the AQ scores for subjects in Group 3, broken down according to their degree/area of study. We compared students among studying Science (i.e., physics, chemistry, biology, mathematics, computer science, engineering, and medicine), vs. Humanities (i.e., classics, languages, philosophy, and history), vs. Social Sciences (i.e., economics, sociology, political sciences, management and geography). We followed the same classification of degree subject as was used in the UK study. There was a main effect of Degree $(F(2,990)=7.362, p<0.01)$, the science students scoring higher than both social science and humanities students $(p<0.01)$, and social science students scoring higher than humanities students 
Table III. Percent of Subjects in Each Group Scoring at or Above Each AQ Score in Japanese Sample

\begin{tabular}{|c|c|c|c|c|c|}
\hline \multirow[b]{2}{*}{ Score } & \multirow{2}{*}{$\begin{array}{c}\text { Group } 1 \\
\text { AS/HFA }(n=57)\end{array}$} & \multicolumn{2}{|c|}{ Group 2} & \multicolumn{2}{|c|}{ Group 3} \\
\hline & & Males $(n=103)$ & Females $(n=91)$ & Males $(n=555)$ & Females $(n=495)$ \\
\hline 5 & 100.0 & 100.0 & 100.0 & 100.0 & 100.0 \\
\hline 6 & 100.0 & 99.0 & 100.0 & 100.0 & 99.8 \\
\hline 7 & 100.0 & 99.0 & 100.0 & 100.0 & 99.2 \\
\hline 8 & 100.0 & 98.1 & 97.8 & 99.8 & 98.6 \\
\hline 9 & 100.0 & 97.1 & 96.7 & 99.3 & 97.2 \\
\hline 10 & 100.0 & 94.2 & 93.4 & 98.0 & 95.6 \\
\hline 11 & 100.0 & 93.2 & 90.1 & 96.6 & 92.9 \\
\hline 12 & 100.0 & 91.3 & 85.7 & 94.4 & 89.7 \\
\hline 13 & 100.0 & 85.4 & 80.2 & 92.1 & 86.5 \\
\hline 14 & 100.0 & 78.6 & 75.8 & 89.0 & 81.2 \\
\hline 15 & 100.0 & 76.7 & 70.3 & 85.2 & 76.2 \\
\hline 16 & 100.0 & 71.8 & 65.9 & 81.6 & 72.3 \\
\hline 17 & 100.0 & 66.0 & 60.4 & 76.9 & 68.1 \\
\hline 18 & 100.0 & 59.2 & 53.8 & 71.5 & 63.4 \\
\hline 19 & 100.0 & 52.4 & 46.2 & 66.3 & 59.0 \\
\hline 20 & 100.0 & 44.7 & 38.5 & 59.5 & 54.3 \\
\hline 21 & 100.0 & 38.8 & 29.7 & 54.8 & 48.9 \\
\hline 22 & 100.0 & 33.0 & 23.1 & 50.1 & 42.8 \\
\hline 23 & 100.0 & 27.2 & 17.6 & 44.0 & 36.2 \\
\hline 24 & 98.2 & 22.3 & 14.3 & 38.0 & 29.9 \\
\hline 25 & 96.5 & 15.5 & 11.0 & 33.9 & 24.6 \\
\hline 26 & 94.7 & 12.6 & 8.8 & 28.8 & 19.4 \\
\hline 27 & 94.7 & 11.7 & 7.7 & 24.3 & 15.6 \\
\hline 28 & 94.7 & 7.8 & 5.5 & 19.8 & 12.3 \\
\hline 29 & 93.0 & 7.8 & 3.3 & 15.5 & 9.5 \\
\hline 30 & 93.0 & 5.8 & 2.2 & 11.4 & 7.1 \\
\hline 31 & 93.0 & 4.9 & 2.2 & 7.6 & 4.6 \\
\hline 32 & 87.8 & 3.9 & 2.2 & 4.9 & 3.0 \\
\hline 33 & 87.8 & 2.9 & 2.2 & 3.6 & 1.8 \\
\hline 34 & 80.7 & 2.9 & 1.1 & 2.3 & 1.2 \\
\hline 35 & 80.7 & 1.9 & 1.1 & 1.4 & 0.4 \\
\hline 36 & 75.4 & 1.0 & 0.0 & 0.9 & 0.0 \\
\hline 37 & 70.2 & 0.0 & 0.0 & 0.7 & 0.0 \\
\hline 38 & 63.2 & 0.0 & 0.0 & 0.5 & 0.0 \\
\hline 39 & 50.9 & 0.0 & 0.0 & 0.4 & 0.0 \\
\hline 40 & 36.8 & 0.0 & 0.0 & 0.4 & 0.0 \\
\hline 41 & 31.6 & 0.0 & 0.0 & 0.2 & 0.0 \\
\hline 42 & 15.8 & 0.0 & 0.0 & 0.2 & 0.0 \\
\hline 43 & 15.8 & 0.0 & 0.0 & 0.2 & 0.0 \\
\hline 44 & 12.3 & 0.0 & 0.0 & 0.2 & 0.0 \\
\hline 45 & 10.5 & 0.0 & 0.0 & 0.2 & 0.0 \\
\hline 46 & 5.3 & 0.0 & 0.0 & 0.2 & 0.0 \\
\hline 47 & 3.5 & 0.0 & 0.0 & 0.0 & 0.0 \\
\hline 48 & 3.5 & 0.0 & 0.0 & 0.0 & 0.0 \\
\hline 49 & 0.0 & 0.0 & 0.0 & 0.0 & 0.0 \\
\hline
\end{tabular}

$(p<0.05)$. There was also a main effect of $\operatorname{Sex}(F(1$, $990)=9.774, p<0.01$ ), males scoring higher than females. There was no significant interaction of Degree by Sex. The science students differed from non-science students on three subscales (social skill and imagination, $t$-tests, $p<0.01$, communication, $p<0.05)$.

\section{Differences Between Types of Sciences}

The four types of sciences contrasted in the UK study (engineering, mathematics, medicine, and physical sciences) by sex were also compared in one ANOVA in the Japanese data. There was a significant main effect of type of science $(F(3,167)=5.631$, $p<0.01)$, mathematics and physical science students 
Table IV. Mean AQ Scores (and SDs) for Students (Group 3) Studying Different Degrees, in Japanese Sample and the UK Study (Baron-Cohen et al., 2001)

\begin{tabular}{|c|c|c|c|}
\hline & & Total AQ in Japanese sample & Total AQ in the UK sample \\
\hline \multicolumn{4}{|l|}{ Sciences } \\
\hline \multirow[t]{2}{*}{ All ( $n=454$ in UK, $n=184$ in Japan) } & $M$ & 22.6 & 18.5 \\
\hline & $S D$ & 6.18 & 6.8 \\
\hline \multirow[t]{2}{*}{ Males ( $n=284$ in UK, $n=127$ in Japan) } & $M$ & 23.1 & 19.3 \\
\hline & $S D$ & 6.07 & 6.8 \\
\hline \multirow[t]{2}{*}{ Females ( $n=170$ in UK, $n=57$ in Japan) } & $M$ & 21.5 & 17.1 \\
\hline & $S D$ & 6.22 & 6.5 \\
\hline \multicolumn{4}{|l|}{ Humanities } \\
\hline \multirow[t]{2}{*}{ All $(n=276$ in UK, $n=631$ in Japan) } & $M$ & 20.1 & 16.7 \\
\hline & $S D$ & 6.32 & 5.9 \\
\hline \multirow[t]{2}{*}{ Males ( $n=119$ in UK, $n=315$ in Japan) } & $M$ & 20.9 & 17.7 \\
\hline & $S D$ & 6.41 & 5.8 \\
\hline \multirow[t]{2}{*}{ Females ( $n=157$ in UK, $n=316$ in Japan) } & $M$ & 19.2 & 15.9 \\
\hline & $S D$ & 6.33 & 5.8 \\
\hline \multicolumn{4}{|l|}{ Social sciences } \\
\hline \multirow[t]{2}{*}{ All ( $n=110$ in UK, $n=181$ in Japan) } & $M$ & 21.0 & 16.4 \\
\hline & $S D$ & 6.71 & 5.8 \\
\hline \multirow[t]{2}{*}{ Males $(n=51$ in UK, $n=105$ in Japan) } & $M$ & 21.8 & 17.1 \\
\hline & $S D$ & 6.82 & 6.1 \\
\hline \multirow[t]{2}{*}{ Females ( $n=59$ in UK, $n=76$ in Japan) } & $M$ & 20.0 & 15.8 \\
\hline & $S D$ & 6.56 & 5.5 \\
\hline
\end{tabular}

scoring higher than two other science students $(p<0.01)$, and engineering students scoring higher than medical students $(p<0.01)$. Mathematicians and physical science students scored significantly higher than all other science students $(p<0.01$ and 0.05 , each), and medical students scored significantly lower than all science students $(p<0.01)$, and medical students scored significantly lower than the mean score of all humanities students $(p<0.10)$. But there were no main effect of Sex and no Science by Sex interaction.

\section{Similarities and Differences Between UK and Japan}

On the whole, the Japanese data was very similar to UK data, although the mean AQ score in Japanese was higher than the British in all three groups by about two points. Comparing the Japanese data and UK data using a $t$-test of the mean total AQ score, in each of corresponding groups, we found that the mean score of the AQ in Japanese data was significantly higher than the UK data, both for controls and students $(t=3.217, p<0.05$ and 10.482, $p<0.01$, respectively). However, the difference between AS/ HFA group and normal groups in the two countries were almost identical: in the UK the mean difference in AQ score between AS/HFA group and controls was 19.4 points, and between AS/HFA group and students was 18.2 points; in Japan they were 19.4 points and 17.2 points, respectively.

Mean subscale AQ scores from each group in both UK and Japan are displayed in Table V. Comparing the UK and Japan data using $t$-tests for each subscale, there were significant differences between UK and Japan on almost all subscales. Though these were found across all three groups, this tendency was clearest in the university student group. Students in Japan scored higher on the "Social skill", "Attention switching", "Communication", and "Imagination" subscales, whereas students in the UK scored higher on the "Local detail" subscale ( $t$-tests, all $p<0.001)$.

\section{DISCUSSION}

This study compared data from the AutismSpectrum Quotient (AQ) in the original UK study with those obtained in a Japanese study. In general, the UK results were replicated in every respect: (1) The AS/HFA group scored at a similar high level (UK cut-off $>32$, Japanese cut-off $>33$ ); (2) A sex difference (males $>$ females) was found in both general populations and student populations, but not among the individuals with AS/HFA; (3) Among the students, scientists scored significantly higher than 
Table V. Mean Subscale Scores (and SDs) of AQ by Group in the UK and Japan

\begin{tabular}{|c|c|c|c|c|c|}
\hline & Social skill & Attention switching & Local detail & Communication & Imagination \\
\hline \multicolumn{6}{|l|}{ Group 1} \\
\hline UK & $7.5(1.9)$ & $8.0(1.8)$ & $6.7(2.3)$ & $7.2(2.0)$ & $6.4(2.1)$ \\
\hline \multirow[t]{2}{*}{ Japan } & $8.3(1.80)$ & $7.9(1.43)$ & $6.2(2.22)$ & $8.2(1.28)$ & $7.4(1.63)$ \\
\hline & $\mathrm{Jpn}>\mathrm{UK}^{*}$ & n.s. & n.s. & $\mathrm{Jpn}>\mathrm{UK}^{* *}$ & $\mathrm{Jpn}>\mathrm{UK}^{* *}$ \\
\hline \multicolumn{6}{|l|}{ Group 2} \\
\hline UK & $2.6(2.3)$ & $3.9(1.9)$ & $5.3(2.3)$ & $2.4(1.9)$ & $2.3(1.7)$ \\
\hline \multirow[t]{2}{*}{ Japan } & $3.4(2.38)$ & $4.3(2.06)$ & $4.9(1.96)$ & $2.8(2.07)$ & $3.2(1.67)$ \\
\hline & $\mathrm{Jpn}>\mathrm{UK}^{* *}$ & $\mathrm{Jpn}>\mathrm{UK}^{*}$ & $\mathrm{UK}>\mathrm{Jpn}^{*}$ & $\mathrm{Jpn}>\mathrm{UK}^{*}$ & $\mathrm{Jpn}>\mathrm{UK}^{* *}$ \\
\hline UK (males) & $2.8(2.5)$ & $4.3(1.9)$ & $5.2(2.3)$ & $2.8(2.0)$ & $2.7(1.9)$ \\
\hline \multirow{2}{*}{ Japan (males) } & $3.7(2.46)$ & $4.2(2.04)$ & $4.7(1.91)$ & $3.0(2.09)$ & $3.4(1.67)$ \\
\hline & $\mathrm{Jpn}>\mathrm{UK}^{* *}$ & n.s. & $\mathrm{UK}>\mathrm{Jpn}+$ & n.s. & $\mathrm{Jpn}>\mathrm{UK}^{* *}$ \\
\hline UK (females) & $2.3(2.2)$ & $3.6(1.8)$ & $5.4(2.3)$ & $2.1(1.8)$ & $1.9(1.5)$ \\
\hline \multirow[t]{2}{*}{ Japan (females) } & $3.1(2.29)$ & $4.3(2.11)$ & $5.0(2.02)$ & $2.6(2.04)$ & $2.9(1.65)$ \\
\hline & $\mathrm{Jpn}>\mathrm{UK}^{* *}$ & $\mathrm{Jpn}>\mathrm{UK}^{*}$ & n.s. & $\mathrm{Jpn}>\mathrm{UK}+$ & $\mathrm{Jpn}>\mathrm{UK}^{* *}$ \\
\hline \multicolumn{6}{|l|}{ Group 3} \\
\hline UK & $2.3(2.2)$ & $4.5(2.0)$ & $5.3(2.2)$ & $2.9(2.0)$ & $2.5(1.9)$ \\
\hline \multirow[t]{2}{*}{ Japan } & $3.9(2.60)$ & $5.2(2.01)$ & $4.8(1.95)$ & $3.7(2.08)$ & $3.2(1.78)$ \\
\hline & $\mathrm{Jpn}>\mathrm{UK}^{* * *}$ & $\mathrm{Jpn}>\mathrm{UK} * * *$ & $\mathrm{UK}>\mathrm{Jpn} * * *$ & $\mathrm{Jpn}>\mathrm{UK} * * *$ & $\mathrm{Jpn}>\mathrm{UK}^{* * *}$ \\
\hline UK (males) & $2.6(2.3)$ & $4.7(2.0)$ & $5.3(2.1)$ & $3.2(2.0)$ & $2.9(2.0)$ \\
\hline \multirow[t]{2}{*}{ Japan (males) } & $4.1(2.57)$ & $5.3(1.96)$ & $4.8(1.96)$ & $3.8(2.11)$ & $3.5(1.86)$ \\
\hline & $\mathrm{Jpn}>\mathrm{UK}^{* * *}$ & $\mathrm{Jpn}>\mathrm{UK}^{* * *}$ & $\mathrm{UK}>\mathrm{Jpn} * * *$ & $\mathrm{Jpn}>\mathrm{UK}^{* * *}$ & $\mathrm{Jpn}>\mathrm{UK}^{* * *}$ \\
\hline UK (females) & $2.0(2.0)$ & $4.3(2.0)$ & $5.4(2.3)$ & $2.7(1.8)$ & $2.0(1.7)$ \\
\hline \multirow[t]{3}{*}{ Japan (females) } & $3.7(2.64)$ & $5.1(2.06)$ & $4.8(1.95)$ & $3.5(2.04)$ & $2.8(1.60)$ \\
\hline & $\mathrm{Jpn}>\mathrm{UK}^{* * *}$ & $\mathrm{Jpn}>\mathrm{UK}^{* * *}$ & $\mathrm{UK}>\mathrm{Jpn}^{* * *}$ & $\mathrm{Jpn}>\mathrm{UK}^{* * *}$ & $\mathrm{Jpn}>\mathrm{UK}^{* * *}$ \\
\hline & $* * * p<.001$ & ${ }^{* *} p<.01$ & $* p<.05$ & $+p<.10$ & \\
\hline
\end{tabular}

non-scientists in both countries, and (4) mathematicians and physicists scientists scored higher than medical scientists in both countries.

These results suggest that autistic conditions at clinical levels of severity are expressed in very similar ways across widely differing cultures. The results also demonstrate the reliability of the AQ in terms of generating very similar patterns of results in otherwise very different samples. The association between $\mathrm{AQ}$ score and scientific ability is also clearly in line with the systemizing theory (Baron-Cohen, 2003; Baron-Cohen, Richler, Bisarya, Gurunathan, \& Wheelwright, 2003): an identical result in both cultures was that a talent for the mathematical or physics end of science is particularly associated with a higher number of autistic traits. These results are also consistent with the extreme male brain (EMB) theory (Baron-Cohen \& Hammer, 1997; Baron-Cohen 2002): sex differences in autistic traits may be a universal characteristic.

In the Japanese sample, just as in the original UK sample, females with AS/HFA appeared to have higher AQs than males with AS/HFA, but in both cultures, males and females with AS/HFA did not differ statistically on the AQ. The mean score in
Japan was slightly higher than the British, but this should not be taken to suggest that AS/HFA is more common in Japan, because the percentage in the general population in both countries scoring at each level on the AQ was remarkably similar. Although some differences were seen on the AQ between the UK and Japan, we emphasis that there are more similarities than differences between the two cultures.

One issue is whether autistic traits in the normal population and those in the autistic spectrum conditions have the same meaning. We think the AQ may well measure autistic traits similarly both in people with/without autistic spectrum. This question could be further investigated by looking at related personality dimensions in both people with and without autism spectrum conditions. Finally, there is an issue whether a quantitative measure of autistic traits is identical to a quantitative measure of AS/HFA. These two goals seem to coincide most of the time, but not always identical. Further studies to investigate this fundamental issue are required.

We conclude that the AQ can be in research studies across different cultures. In clinics where adults are to be assessed for a very late diagnosis of AS or HFA it can also be useful to use the AQ as a 
screening instrument, in order to gauge the appropriateness of the referral and thereby use clinic time effectively. Whether the use of the AQ as a population screening instrument would be warranted will depend on population-wide studies with this instrument, and on the benefits of screening in an adult population. We reiterate that the AQ is not diagnostic, but a screening instrument with clear value in research and clinical settings. A child version of the AQ has been tested in the UK (Baron-Cohen, Hoekstra, Knickmeyer, \& Wheelwright, submitted), and it would be of interest to test this cross-culturally too.

\section{ACKNOWLEDGMENTS}

SBC and SW were supported by a grant from the MRC during the period of this work. We are grateful to the individuals with high-functioning autism and Asperger Syndrome and their parents for their participation. Finally, we thank Professors K Narita, S Tsukamoto, H Yamada, and Mr. A Senju for their help with data collection.

\section{REFERENCES}

APA. (1994). DSM-IV diagnostic and statistical manual of mental disorders (4th ed.). Washington, DC: American Psychiatric Association.

Bailey, T., Le Couteur, A., Gottesman, I., Bolton, P., Simonoff, E., Yuzda, E., \& Rutter, M. (1995). Autism as a strongly genetic disorder: Evidence from a British twin study. Psychological Medicine, 25, 63-77.

Baron-Cohen, S. (1995). Mindblindness: An essay on autism and theory of mind. Boston: MIT Press/Bradford Books.

Baron-Cohen, S. (2002). The extreme male brain theory of autism. Trends in Cognitive Sciences, 6, 248-254.

Baron-Cohen, S. (2003). The essential difference: Men, women and the extreme male brain. London: Penguin.

Baron-Cohen, S., \& Hammer, J. (1997). Is autism an extreme form of the male brain? Advances in Infancy Research, 11, 193-217.

Baron-Cohen, S., Hoekstra, R., Knickmeyer, R., \& Wheelwright, S. (submitted). The Autism-Spectrum Quotient (AQ) - children's version.

Baron-Cohen, S., Richler, J., Bisarya, D., Gurunathan, N., \& Wheelwright, S. (2003). The Systemising Quotient (SQ): An investigation of adults with Asperger syndrome or high functioning autism and normal sex differences. Philosophical Transactions of the Royal Society, Series B, Special issue on "Autism: Mind and Brain", 358, 361-374.

Baron-Cohen, S., Wheelwright, S., Skinner, R., Martin, J., \& Clubley, E. (2001). The Autism Spectrum Quotient (AQ): Evidence from Asperger syndrome/high functioning autism, males and females, scientists and mathematicians. Journal of Autism and Developmental Disorders, 31, 5-17.

Frith, U. (1991). Autism and Asperger's syndrome. Cambridge: Cambridge University Press.

Rutter, M. (1978). Language disorder and infantile autism. In: M. Rutter, \& E. Shopler (Eds.), Autism: A reappraisal of concepts and treatment. New York: Plenum Press.

Wing, L. (1981). Asperger syndrome: A clinical account. Psychological Medicine, 11, 115-130.

Wing, L. (1988). The autistic continuum. In: L. Wing (Ed.), Aspects of autism: Biological research. London: Gaskell/Royal College of Psychiatrists.

Wing, L., \& Gould, J. (1979). Severe impairments of social interaction and associated abnormalities in children: Epidemiology and classification. Journal of Autism and Developmental Disorders, 9, 11-29.

World Health Organization. (1992). International classification of diseases (10th ed.). Geneva, Switzerland: World Health Organisation. 